\title{
Unsichere Sicherheit und Sichere Unsicherheit - Gedanken zu einer "neuen Normalität" im Bildungsbereich mit globaler Perspektive
}

\begin{abstract}
Zusammenfassung
Im Spannungsfeld von Weltgesellschaft und Weltgemeinschaft werden zentrale Phänomene und Zusammenhänge der Transformation für Kommunikation und Handlungsoptionen zwischen „alter“ und „neuer“ Normalität beschrieben. Ausgehend von Reflexionsofferten Globalen Lernens werden mögliche Wandlungsschritte von Bildungseinrichtungen, Didaktik und Lehrenden-Professionalität in ihrer historisch-systematischen Bedeutung für Global Citizenship Education angedeutet.
\end{abstract}

Schlüsselworte: Sicherheit, Normalität, Transformation, Weltgesellschaft, Weltgemeinschaft, Global Citizenship Education

\begin{abstract}
Within the tension of world society and world community, the articel describes central phenomena and connections of the transformation for communication and options for action between "old" and "new" normality. On the basis of offers for reflection based in global learning, possible changes in educational institutions, didactics and teaching professionalism are indicated in their historical-systematic significance for global citizenship education.
\end{abstract}

Keywords: security, normalcy, transformation, world society, world community, Global Citizenship Education

\section{Plötzlich alles anders im Alltag!?}

Seit dem Beginn der Covid-19-Pandemie hat sich der kommunikative Austausch verändert. Ich bekomme und schreibe unzählige E-Mails, erhalte stetig steigende Angebote von Online-Workshops, bin über WhatsApp mit Freund/-innen in aller Welt verbunden und verbringe viel Zeit in Online-Meetings - sowohl lokal, regional, national als auch international. Ich bin seit März 2020 virtuell noch stärker international mit Kolleg/-innen verbunden. Es geht um die Situation nach den Lockdowns. Ich erfahre über verzögerte Auszahlungen zugesagter Hilfsgelder in Japan, verzweifelte und sterbende Menschen auf indischen Straßen, mangelnden Zugang zu Wasser in Brasilien, chilenische Studienbedingungen, Ausgangsbeschrän- kungen in China oder den Muezzin in Ägypten, der Menschen seit Monaten auffordert, daheim zu beten und nicht in die Moschee zu kommen. Ich kann die Berichte als Informationen einordnen und mit Emotionen verbinden, weil ich die Orte kenne, an denen sie relevant sind und die Menschen, die sie berichten. Es sind Narrative des Vertrauten.

\section{Digitalisiertes Lehren und Lernen: Virtuelle Kommunikation und konkrete Optionen!}

Um das Ganze reflexiv einordnen zu können, hilft mir meine jahrelange Beschäftigung mit Globalem Lernen und Global Citizenship Education auf theoretischer und praktischer Ebene. Denn die kommunikative Einheit der Differenz von Sicherheit und Unsicherheit, die sich als Risiko und Variationsvielfalt darstellt, wird in ihrer hohen Komplexität immer greifbarer. Insofern weiß ich darum, dass die virtuellen Erfahrungen lediglich kommunikative Erfahrungshorizonte bleiben, die keinen direkten Spielraum des Handelns bieten. Zugleich habe ich aufgrund der eigenen Erfahrungen mit den Menschen in ihren Lebenswelten in den letzten Jahren eine Idee, welche Handlungsoptionen sich für meine Kolleg/-innen und Freund/-innen bieten, an denen ich kommunikativ partizipieren und einfühlend teilhaben kann. Gleichzeitig weiß ich um die Schwierigkeit, über die gegebene geographische Distanz hinweg in ein konkretes Handeln kommen zu können, zu dem ich etwas beitragen möchte. Deutlich wird in dieser ersten Beschreibung der Unterschied gesellschaftlich-kommunikativer und gemeinschaftlich-interaktiver Räume sozialer Ordnung, die eine hohe Relevanz für Bildungsoptionen und Lernofferten haben - die Weltgesellschaft ist ein kommunikatives Angebot, die im Horizont der Weltgemeinschaft als erfahrbar angenommen werden kann (Lang-Wojtasik, 2013). Dieser Zusammenhang wird in digitalen Settings greifbar.

Denn parallel organisiere ich meine Lehre digital. Glücklicherweise hatte ich mich in den letzten Semestern in Grundzügen mit Optionen digitaler Lehre beschäftigt und konnte auf Erfahrungen eines Projekts international-kooperativer Hochschullehre zum Thema „Global Medial“ zurück- 
blicken, welches mein grundlegendes Verständnis der Medienkompetenz für Globales Lernen geschärft hat (Lang-Wojtasik, Stratmann \& Erichsen-Morgenstern, 2020). Als Studiendekan wurde mir im Austausch mit Kolleg/-innen deutlich, dass es in den meisten Settings eher um DigitalDidaktik, denn ernst zu nehmender Digitaler Medienkompetenz geht, die Medienkritik, -kunde, -nutzung und -gestaltung als interdependente Dimensionen eines Ganzen umfassen müsste (ebd., S. 56 f.). Im Kern des Tuns nahm ich eher war, dass alle irgendwie durchkommen und ein Scheitern vermeiden wollten. So war Vieles einem gewissen Pragmatismus geschuldet, der professionelle Debatten um Medienpädagogik und -didaktik der letzten Jahre eher ausblendete. Immerhin war die Bandbreite digitaler Fähigkeiten und Fertigkeiten breit gestreut. Im Kontakt mit Lehrkräften allgemeinbildender Schulen wurde deutlich, dass sie mit ähnlichen Herausforderungen konfrontiert waren. Gleichwohl ist es beeindruckend, dass es in vielen Fällen durch ausprobierende Kreativität gelungen ist, ein digitales Schuljahr und Semester zu realisieren - wenn auch mit sehr unterschiedlichen Erfolgen. Die Ungleichheit auch in der deutschen Bildungslandschaft v. a. für sozioökomisch schlechter Gestellte ist kein Covid-19-Phänomen. Die ungerechte Verteilung der vorhandenen Güter wurde allerdings pandemiebedingt noch sichtbarer. In der synchron-virtuellen Arbeit mit Studierenden in meinen beiden Vorlesungen (320 und 160 Teilnehmenden) fiel mir auf, dass die meisten vermieden, ihren Bildschirm freizugeben. Als Gründe wurden häufig technische Herausforderungen benannt. Im Verlauf der Veranstaltungen wurde klar, dass eine stattliche Zahl von Studierenden bewusst das Video nicht einschaltete, weil parallel andere Dinge erledigt oder mehrere Meetings gleichzeitig absolviert wurden. Das „Blackboard“ entwickelte für mich als Dozent bei dem Blick in den Orkus schwarzer briefmarkengroßer Namensschilder eine neue Bedeutung. Der Wunsch nach anonymer Dienstleistung im Lehr-Lern-Prozess bekam neue Nahrung. Bei alledem fehlte von Anfang an die soziale Präsenz. Alle mussten sich zurechtruckeln und in der digitalen Welt neu orientieren. Bildungskonzeptionen, die sich mit global-vernetzten Herausforderungen beschäftigen, stehen vor konkreten didaktischen Herausforderungen, die erahnt wurden und plötzlich greifbar sind. Insofern fordert Covid-19 Entwicklungspädagogik, Globales Lernen und Global Citizenship Education massiv heraus (Irish Aid, 2020; Wintersteiner, 2020).

Bildung ist seit jeher eine Chance, als autonom und mündig begriffene Menschen zu strukturellen Änderungen ihrer Weltsicht und -gestaltung zu ermuntern. Global Citizenship Education ist hier ein besonderes Angebot (Lang-Wojtasik, 2019a); wenn es gelingt, den ,vorherrschenden eurozentrischen Denkrahmen“ zu überwinden (Wintersteiner, 2020, S. 1) und das Angebot des Planeten als „Heimatland Erde“ (Morin; zit. n. ebd., S. 6) zu würdigen. Die Gestaltung der Zukunft funktioniert nur gemeinsam. Wir im globalen Norden müssen endlich aufhören, über unsere Verhältnisse zu leben und uns auf den Weg einer aufrichtigen Partnerschaft mit der Mehrheit der Weltbevölkerung machen: Wie wollen wir zusammen und mit dem Planeten leben? „Normalität“ wird bei alledem v. a. in zwei Richtungen relevant - gesellschaftlich als Umgang mit den Herausforderungen des Planeten und hochschuldidaktisch als Erprobung des „digitalen Notfalls“. Ist das alles normal und will ich das als Normalfall? Wenn die Rückkehr zum Bekannten normal ist, was kann dann neue Normalität sein? Wie kann ich soziale Präsenz trotz asozialer Bedingungen herstellen? Wie lässt sich „neues Normales“ (Irish Aid, 2020) bildungsrelevant fassen, um etwas zur zukunftsfähigen Transformation der Erde beizutragen?

\section{Unsichere Sicherheit als „alte Normalität“?}

Möglicherweise ermöglicht der Umgang mit Covid-19 einen Wandlungspunkt im Umgang mit gesellschaftlichen Herausforderungen und damit verbundenen Bildungsoptionen. Die Pandemie ist ein greifbares Warnsignal, wie Menschen miteinander und mit dem Planeten als ihrer gemeinsamen Lebensgrundlage umgehen wollen. Bezüglich gesellschaftlicher Herausforderzungen müsste spätestens seit Beginn und Verlauf der Covid-19-Pandemie auch den letzten Zweifler/-innen im globalen Norden deutlich geworden sein, dass Herausforderungen der Welt nur gemeinsam angegangen werden können. Die Proteste im Rahmen von Fridays for Future weisen regelmäßig darauf hin, dass die im globalen Norden liebgewonnenen „zivilisatorischen Errungenschaften“ nur auf Kosten anderer Menschen und der Überlastung des Planeten möglich sind. Insofern ist es überraschend, dass Covid-19 und die Klimakatastrophe unabhängig voneinander betrachtet werden. Denn das bekannte Zivilisationsmodell ist erkennbar nur wenig tragfähig, um Zukunftsfähigkeit für eine solidarische Weltgemeinschaft möglich zu machen. Ernstgemeinte Solidarität bedeutete Umverteilung des vorhandenen Wohlstandes und eine veränderte Vorstellung von Zivilisation, wie es anschaulich im Rahmen der Sustainable Development Goals (SDGs) beschrieben wird (UN, 2015), die einen partnerschaftlichen Ausgleich zwischen globalem Norden und Süden im Blick haben.

Ob der lieb gewonnene Lebensstil jemals „normal“ war, ist dabei eine zentrale Herausforderung. Wenn Menschen und insbesondere Politiker/-innen heute von einer „,neuen Normalität“ sprechen, ist zu klären, was genau das Neue sein soll und kann. Zudem bleibt unklar, wer in Zukunft die Grundlagen von Normalität bestimmt. Das ist eine Machtfrage, bei der wir im globalen Norden im Sinne der Nachhaltigkeit schlecht aussehen und gleichzeitig handlungsfähiger sein könnten, als wir es oft sind. Wenn wir Werte und Normen haben, kennen wir die Grenzen des Handelns. Es wird meistens eng, wenn es an das eigene Handeln geht. Viele von uns kennen die globalen Statistiken zu den Herausforderungen der Zeit und engagieren sich leidenschaftlich für andere. Dabei ist den meisten vermutlich das Gefühl von Hilflosigkeit ein stetiger Begleiter. Immerhin können die täglichen Meldungen kommunikativ überfordern und es kann einfacher erscheinen, schlicht den Kopf in den Sand zu stecken, als sich mit neuer Kraft in gemeinschaftliche Aktivitäten zu stürzen. Das Ganze ist oft gepaart mit Kraftlosigkeit und dem Wissen darum, dass das eigene Tun wahrscheinlich nur wenig mehr ist als ein Tropfen auf den berüchtigten heißen Stein.

Die folgenden Informationen sind frei zugänglich im Internet verfügbar. Sie haben hier eine illustrierende Funktion. Daher wird auf Details zur Messmethodik o. Ä. verzichtet. Es sind acht Beispiele von Informationen, die vor der globalen Covid-19-Pandemie berichtet wurden. Sie waren Teil der bekannten „alten Normalität“. ${ }^{1}$ 
- Biodiversität: Laut IPBES (Intergovernmental SciencePolicy Platform on Biodiversity and Ecosystem Services) sind 2019 bei gleichbleibendem Lebensstil in den kommenden Jahren und Jahrzehnten Millionen von Arten vom Aussterben bedroht.

- Atomkraft: Derzeit laufen weltweit 442 AKW. Alleine in Deutschland werden sich nach Angaben von Greenpeace bis zum Jahr 202215.000 Tonnen hoch radioaktiver Müll angesammelt haben, für den es kein Endlager gibt.

- Kriege: Nach SIPRI-Angaben gab es 2019 weltweit 32 Kriege.

- Rüstung: Die Militärausgaben betrugen im gleichen Jahr 1,92 Billionen US Dollar. Sie haben sich im Vergleich zum Vorjahr um 3,6 \% erhöht.

- Armut: In Subsahara-Afrika und Südasien lebten 2019 nach UN-Angaben fast $85 \%$ der Bevölkerung in multidimensionaler Armut (der Index umfasst neben dem verfügbaren Geld/Tag auch weitere Indikatoren aus den Bereichen Gesundheit, Bildung und Lebensstandard).

- Verteilung des Wohlstands: Nach einer Studie von Oxfam 2018 strich das reichste Prozent der Menschen 82\% des 2017 erwirtschafteten Vermögenswachstums ein, während die ärmere Hälfte der Weltbevölkerung leer ausging.

- Migration und Flucht: Aktuell sind nach UNHCRAngaben mehr als 70 Millionen Menschen auf der Flucht.

- Rechtsextremismus: Ende 2019 galten mehr als 30.000 Menschen in Deutschland nach Verfassungsschutzangaben als rechtsextrem.

So willkürlich diese ausgewählten acht Themenbereich sein mögen, sie weisen uns auf zentrale Herausforderungen der Menschheit in der fortschreitenden Moderne hin, von denen die meisten spätestens seit Anfang der 1970er bekannt sind und deren Lösung bereits damals als dringlich angesehen wurde (Meadows et al., 1972): Bewahrung der Schöpfung, Ressourcenverteilung und Energieversorgung; Frieden, Sicherheit \& Überwindung der Gewalt; Entwicklung, Verteilung des Wohlstands und Überwindung der Armut; Migration, Interkulturalität und Menschenwürde/-rechte (Lang-Wojtasik, 2020, S. 184 f.).

Genau genommen ist dies jene „alte Normalität“, die immer dann zur Disposition stand, wenn sie mit normativen Forderungen einer „Weltbürgerschaft“ (WBGU, S. 8) oder einer „Weltbürgergesellschaft“ (WBGU, S. 214) verbunden wurde. Die damit verbundene Tradition reicht weit in die Menschheitsgeschichte zurück (Nussbaum, 2020). Wenn heute in politischen Statements, in Radioreportagen, Zeitungsartikeln oder Gesprächen mit Freund/-innen und Kolleg/-innen von einer „Rückkehr zur Normalität" die Rede ist, wird meistens darauf gehofft, zu etwas zurückzukehren, das die angedeuteten Diskrepanzen selbstverständlich umfasst und letztlich ausblendet. Sicherheit als erhoffter Normalfall ist dann auf einen sehr selektiven Weltblick angewiesen, der die weltweiten Zusammenhänge und Verstrickungen gedanklich „parkt“, obwohl die Grundwerte des „aufgeklärten Europa“ auf dem Spiel stehen. Von Zeit zu Zeit lassen dann medial vermittelte Nachrichten aufhorchen und stellen die gewohnte „Normalität“ in Frage. Wenn Menschen noch Nachrichten zur Kenntnis nehmen, müsste auffallen, dass zumindest die Wetterberichte täglich neue Alarmszenarien berichten - zu wenig Niederschläge in den meisten Teilen Deutschlands drei Jahre in Folge und durch Trockenheit sterbende Wälder. Wenn das normal ist, was ist dann anormal? Oder braucht es erst Bilder des brennenden Flüchtlingslagers in Moria, um Menschen aus ihrer selbst beschriebenen Normalität zu reißen, die seit Jahrzehnten anormal ist? Kommunikativ wussten wir alle, dass Griechenland und Italien mit den Hauptherausforderungen der EU-Flüchtlingspolitik konfrontiert sind. Die Bilder der Rohingya-Flüchtlingslager in Bangladesch oder Binnenflüchtlinge im Südsudan flimmerten von Zeit zu Zeit über deutsche Bildschirme und blieben auf Distanz. Was weit weg erscheint, sieht bei genauer Betrachtung ähnlich aus wie Moria oder die anderen unzähligen griechischen Lager. Mich erinnert es immer wieder an slumähnliche Behausungen, die ich aus Indien kenne. Unabhängig davon, um welche Region der Welt es geht - es sind Menschen, die in einer Notlage sind. Das kann uns Bewohner/-innen des globalen Nordens nicht kaltlassen, die wir uns in der Tradition europäischer Aufklärung und eines christlich-jüdischen Abendlandes wähnen. Diese „alte Normalität“ unsicherer Sicherheit erfordert fundamentale Veränderungen im Weltmaßstab, die sich an einer nachhaltigen Entwicklung orientieren und Fragen der Gerechtigkeit und Partnerschaft aufrichtig neu vermessen müssen. Was heißt dabei Solidarität angesichts fast unbegreifbarer Unübersichtlichkeit? Wie lässt sich dies durch bildungsbezogene Maßnahmen und Angebote rahmen, damit sinnvolles Handeln als machbar angenommen werden kann?

\section{Sichere Unsicherheit als „neue Normalität“?}

Aus der Perspektive Globalen Lernens war und ist es möglich, die soziologisch generierte und philosophisch anschlussfähige Figur der Weltgesellschaft (Luhmann, 1997, S. 145 ff.) als Kommunikation über nationalstaatliche und -gesellschaftliche Grenzen hinweg zu beschreiben und systematisch auf Bildungsfragen zu beziehen (Treml, 2000; Seitz, 2002): Entgrenzung, Glokalisierung und Vernetzung (räumlich); Entzeitlichung und beschleunigter sozialer Wandel (zeitlich); zunehmende Komplexität von Informationen und wachsende Kontingenz legitimierbarer Entscheidungen (sachlich); Individualisierung und Pluralisierung von Personen und ihren Lebenswelten (sozial) (Treml, 2000; Scheunpflug, 2011; Lang-Wojtasik, 2013).

Die Weltgesellschaft umfasst kommunikativ alles Denkbare. Die damit verbundene Grenzenlosigkeit ist konsequenterweise die Grenze des Vorstellbaren. In diesem Rahmen geht es um Überschaubarkeit im Umgang mit Offenheit und Entgrenzung (räumlich). Dazu gehört auch, Glokalisierung (Robertson, 1998) als ineinander verwobene Differenz globaler und lokaler Phänomene zu begreifen sowie Vernetzung als wachsende Tatsache über bisher grenzziehende nationalstaatliche Grenzen hinweg und Chance neuer Optionen wahrzunehmen. Dieser rahmende räumliche Blick ist eng verwoben mit zeitlichen, sachlichen und sozialen Blickwinkeln. Kommunikative Prozesse finden jenseits begrenzender Zeitzonen statt (Entzeitlichung) und kommen zunehmend als beschleunigt in den Blick (zeitliche Perspektive). Dies hat Konsequenzen für die individuelle Handhabbarkeit kommunikativer Angebote als auch bezogen auf Orientierungspunkte, die sich gesellschaftlich in Werten und Normen manifestieren. Die Beschleunigung des sozialen Wandels erschwert legitimierbare Stand- 
punkte in der Gegenwart mit Blick auf eine unsichere Zukunft und bodengeerdete Vergangenheit. Dies ist eng verbunden mit den Herausforderungen in sachlicher Perspektive. Die Menge verfügbarer Informationen - ob redundant sei dahingestellt erscheint als exorbitant steigend. Die Beschreibbarkeit von Zusammenhängen ist aufgrund wachsender Komplexität als immer schwieriger. Damit steigt auch die Kontingenzproblematik. Immerhin müssen sich Menschen für und damit gegen etwas anders entscheiden und dies legitimieren können, um im Moment handlungsfähig zu bleiben. Schließlich verändert sich die Wahrnehmbarkeit sozialer Phänomene und Zusammenhänge. Immerhin kommt die zentrale Errungenschaft der europäischen Moderne, den Menschen als autonomes, freies Individuum zu begreifen, als nicht endender Individualisierungsprozess in den Blick. Wenn die Individualisierung der Vielen weitergedacht und als gleiche Verschiedenheit für alle begriffen wird, ist Pluralisierung der Einzelnen im Kollektiv der anderen der Normalfall (Treml, 2000). Diese Weltgesellschaft fordert Menschen massiv heraus, wenn es um Optionen des Machbaren geht. Immerhin verändert sich der bisher rahmengebende kommunikative Referenzrahmen für Entscheidungen unter der wachsend wahrnehmbaren Prämisse von Unsicherheit, Risiko und Variationsreichtum. Anthropologisch ist es wenig überraschend, dass Menschen auf Sicherheit, Abschätzbarkeit von Gefahr und Handhabbarkeit angewiesen sind, wenn es um die Gestaltung ihrer konkreten Umwelt geht (Lang-Wojtasik, 2017a). Damit sind gesellschaftliche und auch pädagogische Fragen eng verbunden. Die angedeutete unsichere Sicherheit der alten Normalität müsste einer sicheren Unsicherheit weichen, mit der anerkannt wird, dass eine Rückkehr zur bisherigen Normalität keine Perspektive hat. Vielmehr müsste die bisher ausgeblendete Anormalität eines lieb gewonnenen Lebensstils im Sinne einer „neuen Normalität“ überwunden werden, die sich als Paradoxien zuspitzen lassen: Begrenzende Entgrenzung (räumlich), Gewisse Ungewissheit (zeitlich), Gewusstes Nichtwissen (sachlich), Vertraute Fremdheit (sozial). In diesem Sinne ist es dann auch möglich, gesellschaftliche Herausforderungen als Ausgangspunkte lebenslanger Bildungsarbeit und Lernanlässen zu begreifen. Individuen brauchen die Gemeinschaft der anderen, um sich über Orientierungspunkte zum Umgang mit sicherer Unsicherheit als „neuer Normalität“ auszutauschen. Ob dabei digitale Formate ausreichen können, wird sich zeigen. Mit Rückblick auf das letzte halbe Jahr würde ich erfahrungsbezogen sagen, dass virtueller Austausch keine soziale Präsenz ersetzen kann und dass alle menschlichen Sinne notwendig sind, um als Mensch agieren zu können. Konkret: Über Nachhaltigkeit und Gerechtigkeit kann ich mich kommunikativ in einem virtuellen Raum austauschen und ich kann über Erfahrungen berichten. Wenn es um weitergehende und vertiefte Perspektiven gehen soll, machen konkrete gemeinsame Erprobungen und Austauschprozesse Sinn.

\section{Global Citizenship Education konkretisieren}

Die Welt, in der wir leben, befindet sich in umfassenden Wandlungsprozessen, die auch als Große Transformation bezeichnet werden (WBGU, 2011). Diese sind folgenreich für die gesamte Menschheit. Neben Fragen von Transformationsforschung (Rahmenbedingungen strukturellen Wandels) und transformativer Forschung (Veränderungsoptionen struktu- rellen Wandels) werden seit geraumer Zeit Transformationsbildung und transformative Bildung unterschieden (WBGU, 2011, S. 23f.; Lang-Wojtasik, 2019a). Jenseits der berechtigten Frage, ob Bildung und Lernen nicht per se transformativ sind (Scheunpflug, 2019), kann Transformationsbildung als der institutionelle und organisatorische Rahmen von Bildung beschrieben werden (Bildungseinrichtung), in der Zukunftsfähigkeit möglich ist. Transformative Bildung lässt sich demgegenüber als das Gesamt zukunftsfähiger Lernofferten begreifen, für das Globale Bildung und Globales Lernen auch im europäischen Sinne (O'Loughlin \& Wegimont, 2003) bedeutsam sind. Globales Lernen ist auch ein wichtiger Wegbereiter für Global Citizenship Education (Wintersteiner et al., 2014). Weitere konzeptionelle Zugänge sind Bildung für nachhaltige Entwicklung und Umweltbildung, Friedenspädagogik und Gewaltfreie Konfliktbearbeitung, Interkulturelle und differenzsensible Pädagogik sowie Menschenrechtsbildung. Jenseits der je eigenen Disziplingeschichten und Zusammenhänge unterliegen ihnen zentrale normative Offerten, die orientierenden Charakter für zukunftsfähige Bildungsarbeit haben: Nachhaltigkeit und wertschätzender Umgang mit dem Planeten, Gleichwürdigkeit und Gerechtigkeit im Umgang miteinander, Partnerschaftlichkeit als konstantes Bemühen solidarischen Lebens und Gewaltfreiheit als Grundlage des Friedens (LangWojtasik, 2019b). Die Konzentration auf Citizenship ermöglicht einerseits eine systematische Verankerung in Anliegen politischer Bildungsarbeit (Wintersteiner et al., 2014) als auch Bezüge zu weltbürgerlicher Erziehung (Treml, 2011). Dies ist anschlussfähig an den vierten Zielbereich der SDGs, in dem die Umsetzung inklusiver, gleichberechtigter und hochwertiger Bildung gefordert und explizit auf Bildung für nachhaltige Entwicklung in der hier beschriebenen Semantik einer Global Citizenship Education verwiesen wird. Vor diesem Hintergrund setzt zukunftsfähige Bildungsarbeit die Realisierung einer Bildung für alle voraus, wie sie Jomtien, Dakar und Incheon immer wieder gefordert wurde (UNESCO, 2020).

Was visionär klingen mag, braucht eine Bereitschaft, sich im Jetzt auf Zukunftsszenarien strukturellen gesellschaftlichen Wandels durch zukunftsfähige Bildungsarbeit einzulassen. Neben Narrativen der Hoffnung ist kontinuierlicher öffentlicher Dialog über die Möglichkeiten und Grenzen der Zukunftsfähigkeit nötig. Dabei ist es weniger eine Frage, ob Solidarität auf Augenhöhe sofort gelingen kann, sondern vielmehr, dass sie alternativlos ist, wenn die Weltgemeinschaft eine Überlebensperspektive haben soll. Kritische Orientierung an historischen Vorbildern eines aufgeklärten Weltbürger/-innentums sowie konzeptionellen Offerten einer damit assoziierten Global Citizenship Education bieten einen Sinn gebenden Rahmen.

Es ist an der Zeit, Bildung wieder neu zu denken und ihr Potenzial in ihren Grundfesten zu respektieren. Das bedeutet z. B., die 6000 Jahre alte Schulgeschichte als funktionale Basis eines Nachdenkens über Schulentwicklung mitzudenken (Lang-Wojtasik, 2018) und auf Optionen lebenslanger Bildungsangebote im Sinne des EFA-Prozesses zu beziehen (UNESCO, 2020). Notwendig erscheint auch, sich Gedanken über die Tragweite einer Revitalisierung Allgemeiner Didaktik in weltbürgerlicher Tradition zu machen und das Transformationspotenzial von Bildung und Lernen pädagogisch und didaktisch in der Weltgesellschaft zu klären (Scheunpflug, 2019; 
Lang-Wojtasik, 2019b). Glokale Abstraktion (räumlich), Entschleunigte Positionierung (zeitlich), Exemplarische Interkulturalität (sachlich) sowie Kooperative Pluralität (sozial) (im Anschluss an Scheunpflug, 2011: Lang-Wojtasik, 2019b, S. 39f.) bieten einen Diskussionsrahmen, der empirisch und theoretisch weiter exploriert werden muss. Bei alledem ist es auch an der Zeit, Professionalität wieder mit einem pädagogischen Ethos zu verbinden, das Kompetenz auf der Grundlage von Mündigkeit begreift, die kognitive, volitionale, motivationale, soziale und moralische Aspekte umfasst, um variable Problemlösungen in Performanz möglich zu machen. Es würde bedeuten, Global Teacher als Global Learner und Facilitator zu begreifen, die sich von der Erwartung des Allwissenden verabschieden und zum Lernbegleitenden verändern wollen, um als change agents agieren zu können (Bourn, 2016; Lang-Wojtasik, 2017b). Sie müssten dazu einen geerdeten Weltblick mit Menschlichkeit verbinden können (räumlich), Entwicklung und Entdeckung zur Grundlage ihres Tuns machen (zeitlich), epochaltypische Schlüsselprobleme als interdependente Querschnittsthemen der Weltgemeinschaft ins Zentrum rücken (sachlich) sowie ihr professionelles Können mit einer Haltung des Respekts und dem dialogischen Blick für den und die andere zu verbinden (sozial) (Lang-Wojtasik, 2017b).

Wenn es gelingt, das transformative Potenzial von Bildung für alle in lebenslanger Perspektive, mit revitalisierter Didaktik im Sinne der Global Citizenship Education sowie der pragmatischen Vision von Global Facilitators zu verbinden, eröffnen sich neue Horizonte zur Verabschiedung „alter Normalität". Zugleich deutet sich eine „Willkommenskultur" zum Umgang mit sicherer Unsicherheit als resilientem Ausgangspunkt „neuer Normalität“ für Weltbürger/-innentum an. Worauf warten wir noch? Machen wir uns auf den Weg!

\section{Anmerkungen}

1 Biodiversität: https://www.spiegel.de/wissenschaft/natur/artensterben-uno-berich t-beschreibt-dramatischen-verlust-der-artenvielfalt-a-1265482.htm Atomkraft: https://www.planet-schule.de/wissenspool/tschernobyl/inhalt/hintergr und/atomkraftwerke-weltweit.html; https://www.greenpeace.de/themen/energiewende/atomkraft/atommuell

Kriege, Rüstung: https://www.sipri.org/sites/default/files/2019-11/yb19_summa ry_de.pdf; https://de.statista.com/statistik/daten/studie/157935/umfrage/laende r-mit-den-hoechsten-militaerausgaben/

Armut: https://www.spiegel.de/politik/ausland/armut-neue-uno-studie-zeigt-wer -wie-arm-ist-a-1276915.html

Verteilung des Wohlstands: https://www.oxfam.de/ueber-uns/aktuelles/2018-01-2 2-82-prozent-weltweiten-vermoegenswachstums-geht-reichste-prozen

Migration und Flucht: https://www.unhcr.org/dac he/31634-weltweit-erstmalsmehr-als-70-millionen-menschen-auf-der-flucht.html

Rechtsextremismus: https:/www.verfassungsschutz.de/de/arbeitsfelder/af-rechtsext remismus/zahlen-und-fakten-rechtsextremismus

\section{Literatur}

Bourn, D. (2016). Teachers as agents of social change. International Journal of Development Education and Global Learning, 7(3), 63-77. https://doi.org/10.18546/IJD EGL.07.3.05

Irish Aid (2020). Covid-19 and Development Education. Policy \& Practice. Development Education Review. Zugriff am 21.9.2020 https://www.developmenteducati onreview.com/issue/issue-30/covid-19-and-development-education

Lang-Wojtasik, G. (2013). Die Weltgesellschaft und der Mensch im Sozialen Wandel. Differenzpädagogische Überlegungen im Horizont von Systemtheorie und Philosophischer Anthropologie. In S. Hornberg, C. Richter \& C. Rotter (Hrsg.), Erziehung und Bildung in der Weltgesellschaft. Festschrift für Christel Adick (S. 13-34). Münster u. a.: Waxmann.

Lang-Wojtasik, G. (2017a). Globalisierung und Globales Lernen als ein Epizentrum politischer Erwachsenenbildung!? Ulm: Klemm \& Oelschläger.

Lang-Wojtasik, G. (2017b). Global Teacher. In G. Lang-Wojtasik \& U. Klemm (Hrsg.), Handlexikon Globales Lernen (S. 198-203). Ulm: Klemm \& Oelschläger.
Lang-Wojtasik, G. (2018). Schulen in der Einen Welt - Perspektiven einer global orientierten Schulentwicklungsforschung. In A. Scheunpflug, H. Simojoki \& M. Schreiner (Hrsg.), Evangelische Schulen und religiöse Bildung in der Weltgesellschaft. Die Bamberger Barbara-Schadeberg-Vorlesungen (S. 97-117). Münster u. a.: Waxmann.

Lang-Wojtasik, G. (Hrsg.) (2019a). Bildung für eine Welt in Transformation. Global Citizenship Education als Chance für die Weltgesellschaft. Opladen u. a.: Barbara Budrich. https://doi.org/10.2307/j.ctvpbnpz8

Lang-Wojtasik, G. (2019b). Globales Lernen als transformative Bildung - Überlegungen zu einer Allgemeinen Didaktik mit weltbürgerlichem Anspruch. In G. Lang-Wojtasik \& S. König (Hrsg.), Die Vielfalt methodischer Zugänge in der Unterrichtsforschung. Beispiele aus Fachdidaktik und Allgemeiner Didaktik (S. 23-48). Ulm u. Münster: Klemm \& Oelschläger.

Lang-Wojtasik, G. (2020). Bildung für Nachhaltigkeit und oder nachhaltiges Lernen? Erziehungswissenschaftliche Überlegungen für Global Citizenship Education. In G. Bade, N. Henkel \& B. Reef (Hrsg.), Politische Bildung: vielfältig - kontrovers - global. Festschrift für Bernd Overwien (S. 181-203). Schwalbach/Ts.: Wochenschau.

Lang-Wojtasik, G., Stratmann, J. \& Erichsen-Morgenstern, R. M. (2020). Online course: "Global Medial" - Global learning through media competence and vice versa. International Journal of Development Education and Global Learning, 12(1), 5268. https://doi.org/10.14324/IJDEGL.12.1.05

Luhmann, N. (1997). Die Gesellschaft der Gesellschaft. Frankfurt a. M.: Suhrkamp. Meadows, De., Meadows, Do., Zahn, E. \& Milling, P. (1972). Die Grenzen des Wachstums. Bericht des Club of Rome zur Lage der Menschheit. Stuttgart: Deutsche Verlags-Anstalt.

Nussbaum, M. (2020). Kosmopolitismus. Revision eines Ideals. Darmstadt: WBG.

O'Loughlin, E. \& Wegimont, L. (2003). Maastricht Global Education Declaration: European strategy framework for improving and increasing global education in Europe to the year 2015. In E. O'Loughlin \& L. Wegimont (Hrsg.), Global Education in Europe to 2015. Strategy, policies, and perspectives. Outcomes and Papers of the Europewide Global Education Congress Maastricht, The Netherlands, 15.-17. November 2002. Lisbon: North-South Centre of the Council of Europe.

Robertson, R. (1998). Glokalisierung: Homogenität und Heterogenität in Raum und Zeit. In U. Beck (Hrsg.), Perspektiven der Weltgesellschaft (S. 192-220). Frankfurt a. M.: Suhrkamp.

Scheunpflug, A. (2011). Lehren angesichts der Entwicklung zur Weltgesellschaft. In W. Sander \& A. Scheunpflug (Hrsg.), Politische Bildung in der Weltgesellschaft (S. 204-215). Bonn: Bundeszentrale für politische Bildung.

Scheunpflug, A. (2019). Transformatives Globales Lernen - eine Grundlegung in didaktischer Absicht. In G. Lang-Wojtasik (Hrsg.), Bildung für eine Welt in Transformation. Global Citizenship Education als Chance für die Weltgesellschaft (S. 63-74). Opladen u. a.: Barbara Budrich. https://doi.org/10.2307/j.ctvpbnpz8.7

Seitz, K. (2002). Bildung in der Weltgesellschaft. Gesellschaftstheoretische Grundlagen Globalen Lernens. Frankfurt a. M.: Brandes \& Apsel.

Treml, A. K. (2000). Allgemeine Pädagogik. Grundlagen, Handlungsfelder und Perspektiven der Erziehung. Stuttgart u. a.: Kohlhammer.

Treml, A. K. (2011). Globalisierung als pädagogische Herausforderung. Möglichkeiten und Grenzen einer weltbürgerlichen Erziehung. In W. Sander \& A. Scheunpflug (Hrsg.), Politische Bildung in der Weltgesellschaft (S. 190-203). Bonn: Bundeszentrale für politische Bildung.

UN (2015). Transforming our world: the 2030 Agenda for Sustainable Development. New York, 25.9.2015; Zugriff am 5.2.2019 https://sustainabledevelopment.un.org/ post2015/transformingourworld

UNESCO (2020). Inclusion and education. All means all. Global Monitoring Report. Paris: UNESCO.

WBGU (2011). Hauptgutachten: Welt im Wandel. Gesellschaftsvertrag für eine Große Transformation. Berlin: WBGU.

Wintersteiner, W. (2020). Die Covid-19-Krise aus einer Global Citizenship Perspektive. Zugriff am 25.9.2020 https://literatur.report/gastbeitraege/die-covid-19-krise-auseiner-global-citizenship-perspektive/

Wintersteiner, W., Grobbauer, H., Diendorfer, G. \& Reitmair-Juárez, S. (2014). Global Citizenship Education. Politische Bildung für die Weltgesellschaft. Wien: Zugriff am 23.9.2020 https://bimm.at/publikationen/global-citizenship-education/

\section{Gregor Lang-Wojtasik}

Prof. Dr. phil. Dr. habil., Professur für Erziehungswissenschaft/Pädagogik der Differenz an der Pädagogischen Hochschule Weingarten, Grund- und Hauptschullehrer, Studiendekan der Fakultät I und Direktor des Forschungszentrums für Bildungsinnovation und Professionalisierung; Arbeits- und Forschungsschwerpunkte: International und Interkulturell Vergleichende Erziehungswissenschaft (Globales Lernen/Bildung für nachhaltige Entwicklung, Friedenspädagogik, Interkulturelle Pädagogik), Schulentwicklungsforschung (Grundbildung und Schultheorie), Wissenschaftstheorie. 